Maximizing Activity and Stability by

Turning Gold

Catalysis Upside Down: Oxide Particles on Nanoporous

Gold

A. Wichmann, A. Wittstock, K. Frank, M. M. Biener, B. Neumann, L. Maedler, J. Biener, A. Rosenauer, M. Baumer

June 15, 2012

CHEMCATCHEM 
This document was prepared as an account of work sponsored by an agency of the United States government. Neither the United States government nor Lawrence Livermore National Security, LLC, nor any of their employees makes any warranty, expressed or implied, or assumes any legal liability or responsibility for the accuracy, completeness, or usefulness of any information, apparatus, product, or process disclosed, or represents that its use would not infringe privately owned rights. Reference herein to any specific commercial product, process, or service by trade name, trademark, manufacturer, or otherwise does not necessarily constitute or imply its endorsement, recommendation, or favoring by the United States government or Lawrence Livermore National Security, LLC. The views and opinions of authors expressed herein do not necessarily state or reflect those of the United States government or Lawrence Livermore National Security, LLC, and shall not be used for advertising or product endorsement purposes. 


\title{
Boosting activity by turning gold catalysis upside down: oxide particles on nanoporous gold
}

\author{
Andre Wichmann ${ }^{1}$, Arne Wittstock ${ }^{1,2^{*}}$, Kristian Frank ${ }^{4}$, Monika Biener ${ }^{2}$, Björn \\ Neumann ${ }^{1}$, Lutz Mädler ${ }^{3}$, Jürgen Biener ${ }^{2}$, Andreas Rosenauer ${ }^{4}$, Marcus Bäumer ${ }^{1^{*}}$ \\ 1 Institute of Applied and Physical Chemistry, University of Bremen, Leobener Str. NW2, 28359 \\ Bremen, Germany \\ 2 Nanoscale Synthesis and Characterization Laboratory, Lawrence Livermore National Laboratory, \\ Livermore, California, USA \\ ${ }^{3}$ Institut für Werkstofftechnik, University of Bremen, Badgassteiner Str. 3, 28359 Bremen, Germany \\ ${ }^{4}$ Institute of Solid State Physics, University of Bremen, Otto-Hahn-Allee 1, 28359 Bremen, Germany
}

*Corresponding authors: mbaeumer@uni-bremen.de, awittstock@uni-bremen.de

\section{Abstract}

Oxidation catalysis by $\mathrm{Au}$ spurred intensive research efforts during the last two decades. It is the intense interaction of gold and an oxide material which brings about the catalytic activity, in particular at the perimeter between both partners. The classical design of highly active and durable catalysts has been impeded by the tendency of gold nanoparticles to sinter, forming larger aggregates with drastically lower activity. We demonstrate that this impediment can be overcome by reversing the classical order, depositing oxide nanoparticles on a high surface area gold support. We employed atomic layer deposition as well as liquid phase deposition leading to densely arranged oxide nanoparticles on the surface of a nanoporous gold material. The dense packing of oxide nanoparticles results in a maximized interface region of both partners, not achievable for gold nanoparticle based catalysts so far. In particular, in the case of a titania coated material, an oxidation catalyst with so far unprecedented high catalytic activity could be obtained. Concomitantly, the metal oxide stabilizes the $\mathrm{Au}$ nanostructure up to $600^{\circ} \mathrm{C}$, opening the door for high temperature applications. 
Heterogeneous catalysis is a key technology not only with respect to the production of basic and advanced materials and chemicals but also with respect to the ever increasing energy and environmental concerns. Gold as a superior catalytic material was discovered about two decades ago and fueled intensive research efforts as it shows higher activity and better selectivity than any other precious metal, particularly at temperatures well below $100{ }^{\circ} \mathrm{C} .(1,2)$ Numerous studies under well-controlled conditions, such as high-vacuum conditions, revealed comprehensible reaction mechanisms rendering the surface chemistry of gold "predictable".(3, 4) This special feature of gold is the result of a "medium" interaction with adsorbates, enabling high activity at low temperatures and highly selective surface reactions.(5)

A general problem of pure $\mathrm{Au}$ in partial oxidation reactions, playing a particularly important role in industrial gas phase catalysis(6), however, is (a) the activation/dissociation of oxygen being not possible on pure gold,(7) and (b) the limited thermal stability of nanosized Au particles, resulting in an insufficient longterm stability due to sintering(8). Accordingly, gold catalysis depends on a team work: Classical heterogeneous Au catalysts need to be in contact with a suitable reducible metal oxide (mostly titania or ceria) serving as a support material and stabilizing the Au nanoparticles. $(9,10)$ In a recent publication, Green et al. provide further insight into the particular interaction between both partners, resulting not only in common adsorption sites for $\mathrm{CO}$ but also enabling activated molecular oxygen to be directly delivered at the interface.(11) As the interface region between the gold nanoparticle and the metal oxide is undoubtedly a crucial factor for highly active gold based catalysts, the interface region between both partners has to be maximized in order to generate highly active catalysts. Unfortunately, the areal density of gold nanoparticles on the metal oxide support is severely limited by the strong tendency to sinter, forming larger aggregates with drastically lower activity.

A simple solution to this problem is to reverse the classical situation, employing a catalytic system, comprised of metal-oxide nanoparticles on a high surface area gold support. As the melting temperature of metal oxides, such as titania, is nearly two times higher than that of gold, the corresponding metal oxide nanoparticles are considerably more stable against sintering. Such an approach, however, has not been considered so far in view of a lacking gold support. 
An interesting material that fulfills the requirement of exhibiting a sufficiently high surface area is nanoporous gold which attracted increasing interest as a catalytic material only recently.(12, 13) This gold foam consists of interconnected gold ligaments and pores in the order of some tenths of nanometers, typically $30-40 \mathrm{~nm}$, thus providing a high specific surface area in the range of $10 \mathrm{~m}^{2} / \mathrm{g}$. A particular advantage of this catalytic material is the stable monolithic structure, combining mechanical strength, thermal and electrical conductivity and a reproducible porosity due to self-organization during the preparation by corrosion (wet chemical leaching of $\mathrm{Ag}$ in an Ag-rich AuAg alloy) with or without potentiostatic control. This material can be prepared in various forms ranging from thin films suitable for coatings with thicknesses as thin as $100 \mathrm{~nm}$, to millimeter sized pieces in the form of cylinders or cubes.(14, 15) The typical bicontinuous nanoporous structure emerges absolutely homogeneously during the preparation even for samples beyond the order of several millimeters. In summary, nanoporous gold appears to be an ideal basis for an inverse catalyst design, with far-reaching options to control structure (porosity) and surface chemistry.

In view of this potential, we addressed the following questions in the current study: Can the scope of catalytic applications for npAu be expanded by combining it with metal oxides in an easy \& reproducible fashion? In particular, in case of oxidation catalysis, can the activity be enhanced - even beyond anything achieved by classical catalyst designs so far - as anticipated by creating large interface areas between the gold support and the metal oxide particles? In addition, how can the nanostructure be conserved even at temperatures of several hundred degrees being not accessible by classical gold nanoparticle catalysts at all?

In search of a good partner for the nanoporous gold substrate we chose $\mathrm{TiO}_{2}$; it is the most investigated metal oxide for gold nanoparticle catalysts, resulting in highly active oxidation catalysts and it is also an interesting material (combination) with respect to photo catalysis.(16) The crucial impediment for conformal and reproducible deposition of surfactants onto the surface of highly porous nano materials such as npAu is the associated high aspect ratio of the pores, inducing mass transport limitation. Atomic layer deposition (ALD) which consists of selflimiting surface reactions of suitable precursors was shown to be superior to other vapor phase deposition techniques in this respect.(17) We accordingly employed this 
technique to generate conformal coatings of titania inside the npAu material as described in ref. (18). However, to demonstrate the generality of this approach we also used wet chemical impregnation of npAu with a viable precursor (titanium isopropoxide, TTIP) followed by a subsequent calcination step generating metaloxide coatings inside the npAu structure as well. (For further details regarding the preparation of samples see supporting online material.)

Both techniques result in densely arranged metal oxide nanoparticles on the inner surface of the npAu material. While the TTIP impregnation leads to particle growth right away, ALD results in closed films first, which have to be cracked up into particles by a subsequent heating step (see supporting online material). Highresolution transmission electron microscopy (HR-TEM) (Figure 1c and 1f) reveal Au ligaments abundantly covered by approximately $5 \mathrm{~nm}$ to $10 \mathrm{~nm} \mathrm{TiO}_{2}$ particles. Further evidence of a dense and homogeneous $\mathrm{TiO}_{2}$ nanoparticle dispersion is provided by the Ti-maps in Figure 1. They also reveal that in case of the ALD coated samples besides finely dispersed nanoparticles $\mathrm{TiO}_{2}$ films do still exist (Figure $1 \mathrm{~b}$ ).

Besides being in an amorphous form, titania can exist in different crystallographic phases (anatase - rutile - brookite) with anatase being reported to be most beneficial with respect to catalytic activity.(19-21) The formation of a particular crystallinity is dependent on the microstructure of the initially deposited metal oxide and the chosen temperature.(22) In order to obtain the favored crystalline anatase particles, the as-prepared amorphous metal-oxide coated npAu foam was heat treated at $400{ }^{\circ} \mathrm{C}$ for $60 \mathrm{~min}$ in case of liquid phase doping by TTIP and at $650{ }^{\circ} \mathrm{C}$ for $100 \mathrm{~min}$ in case of ALD modified disks, respectively. The higher temperature in the latter case is associated with the mentioned breakup of the initially closed layer of titania on the npAu surface which does not occur at temperatures below about 650 ${ }^{\circ} \mathrm{C}$.(23) The crystallinity of the $\mathrm{TiO}_{2}$ nanoparticles was studied by Raman spectroscopy (cf. Figure S3) and HR-TEM. The determination of the largest lattice fringe distance with HR-TEM (see the lattice fringe intensity scan along the indicated line in Figure 1d) proved that the crystalline nanoparticles indeed consist of anatase. The lattice plane of $0.36 \pm 0.01 \mathrm{~nm}$ is in a good agreement to the literature(24) $(0.35$ $\mathrm{nm})$ for $\{101\}$ lattice planes of this phase. 
The catalytic activity of pure and titania coated npAu samples for the aerobic oxidation of $\mathrm{CO}$ was measured in a continuous flow reactor at various temperatures, starting at reactor temperatures of $40{ }^{\circ} \mathrm{C}$ up to $300{ }^{\circ} \mathrm{C}$ (cf. Figure 2b). The CO conversion of the pure npAu catalyst decreases with increasing temperature which is in line with coarsening of the Au ligaments and the concomitant loss of active surface area (cf. Figure S5). In contrast, the $\mathrm{CO}$ conversion of the $\mathrm{TiO}_{2}$ coated foams increased with increasing temperature showing a steep jump of activity between 60 ${ }^{\circ} \mathrm{C}$ and $100{ }^{\circ} \mathrm{C}$ and subsequently increasing in a parabolic fashion indicating that mass transport (Knudsen Diffusion) in and out of the pores is rate limiting. The activity jump was always found in the same temperature range, independent of the preparation procedure (ALD or liquid TTIP impregnation), indicating a general behavior, such as a mechanistic change at this temperature. This finding is in good agreement with studies of the CO oxidation over gold nanoparticles deposited on titania supports $(25,26)$, for which two mechanistic regimes with a transition around $50{ }^{\circ} \mathrm{C}$ were reported. (In case of the TTIP impregnated sample, the activity jump was observed at somewhat higher temperatures of about $120^{\circ} \mathrm{C}$ in the first run (data not shown here). This indicates an activation process of the surface most likely due to cleaning from adsorbed contaminations.) Noteworthy, due to the monolithic and heat conductive structure of the npAu we were able to directly measure the catalyst temperature which as a consequence of the highly exothermic nature of the oxidation reaction is higher than the reactor temperature (cf. Figure S5). On the one hand, this enables us to directly asses the reactivity as a function of the catalyst temperature not being possible in a similar fashion with Au nanoparticles on an oxide support. On the other hand, it is important in the context of homogeneous and effective heat dissipation preventing over-heating being one main obstacle for the stability of gold nanoparticles so far.

In comparison to the "pure" nanoporous gold sample (with residual silver helping to activate oxygen), the activity of the metal oxide coated samples is increased by more than one of order of magnitude in the temperature range above $60^{\circ} \mathrm{C}$. Noteworthy, both deposition methods - TTIP liquid phase impregnation and the ALD coating achieved identical activities above a CO concentration of 10 volume\%. Of course, as shown in a previous study for pure npAu, due to the fine porosity of the deployed free standing npAu films the observable conversion is strongly limited by diffusion of 
the reactants into pores of the material.(27) Taking this kind of mass transport limitation into account (28), a reaction rate can be calculated which is even a factor of 200 higher than for pure npAu. The estimated TOF (turn over frequency: number of molecules converted per time and catalytically active surface atom) is then in the range of $100 \mathrm{~s}^{-1}\left(\sim 8 \mathrm{mmol} /\left(\mathrm{s} \cdot \mathrm{g}_{\mathrm{cat}}\right)\right)$ at a sample temperature of $100{ }^{\circ} \mathrm{C}$ (while it is in the range of $2-7 \mathrm{~s}^{-1}\left(0.1-0.55 \mathrm{mmol} /\left(\mathrm{s} \cdot \mathrm{g}_{\mathrm{cat}}\right)\right)$ at lower temperatures resembling the values for pure npAu and also for some highly active Au nanoparticle catalysts (2931)). Since this number only constitutes a lower limit of the TOF for not all Au surface atoms are probably catalytically active, this is a remarkable number clearly exceeding all values reported so far for gold catalysts.(31, 32)

In search of other oxide partners for the nanoporous gold substrate enhancing its oxidation capabilities, we also studied praseodymia which is a less common support, but known for its rich redox chemistry. In contrast to titania, a simpler preparation approach was chosen. A $\operatorname{Pr}\left(\mathrm{NO}_{3}\right)_{3} /$ ethanol $(20 \mathrm{~g} / \mathrm{L})$ solution was used for the impregnation carried out for 15 minutes. After drying, the sample was heated (calcined) in air to $500{ }^{\circ} \mathrm{C}\left(7.3^{\circ} \mathrm{C} / \mathrm{min}\right.$ heating rate). The activity of such samples for CO oxidation was somewhat lower than that for titania (factor of 5); this lower performance might be due to the less efficient coating, as revealed by the SEM results (Figure S6). In spite of this, also for praseodymia the activity increase is remarkable (by factor of 4.5 as compared to the pure npAu; activity of the PrOx-npAu sample: $\left.0.1 \mathrm{mmol} /\left(\mathrm{s} \cdot \mathrm{g}_{\mathrm{cat}}\right)\right)$, underlining the generality of the approach to boost the activity of npAu by oxide nanoparticles.

The fact that the activity increases with increasing temperature while it decreases for pure npAu due to coarsening of the ligaments, already implies that not only the activity, but also the thermal stability is drastically improved. Indeed, SEM studies revealed that, while the ligament size of the pure npAu is drastically increased upon annealing, the metal oxide deposits lead to a complete stabilization of the structure. This suppression of coarsening can most likely be explained by pinning of $\mathrm{Au}$ surface atoms, especially at step edges, resulting in a reduction of the surface selfdiffusivity of Au.(8) As shown in Figure 3b/S5, the original npAu structure could be conserved up to temperatures as high as $600{ }^{\circ} \mathrm{C}$ after functionalization. Whereas full stabilization of the nanoporous structure along the whole cross section was achieved after $\mathrm{TiO}_{2}$ deposition with ALD and TTIP respectively, in the case of praseodymia, 
coarsening was suppressed within a layer of $10-15 \mu \mathrm{m}$ close to the outer surface upon heating to $500^{\circ} \mathrm{C}$. Below this layer, a bimodal distribution of stabilized and unstabilized areas was observed (see Figure S6) being probably the result of a limited penetration of the precursors into the narrow and tortuous pores of the material.

For the samples used in this study, such an incomplete deposition is not of importance, since the very inner sections of the sample do not considerably contribute to the catalytic activity due to mass transport limitation; see above. The virtually same conversion of $\mathrm{CO}$ for the ALD and TTIP coated samples is a consequence of the reduced concentration of reactants in these inner sections (27) and thus limited contribution to the overall conversion of CO. Apart from a monolithic samples, free standing films or coatings with thicknesses below $1 \mu \mathrm{m}$ can however be realized by using gold leafs, in all cases with well-defined and reproducible pore structures. Furthermore, applying suitable templating techniques, the relative density (fraction of actual gold per volume) can be reduced from 30 percent for typical free standing films down to 2 percent.(33) Using such thin-walled materials, mass transport limitation can be avoided and the amount of gold material to be used can be minimized, shifting it into the regime of supported catalysts, yet, still having a stable nanostructured corpus.

Both, the significantly improved temperature stability in conjunction with the very high catalytic activity open the door for high temperature applications, such as exhaust gas catalysis. Very recent developments show that gold can be indeed employed in commercial emission control devices as diesel car catalyst (NS Gold ${ }^{\mathrm{TM}}$ by Nanostellar Inc.), underlining the potential of gold's commercialization. In our study using this new type of gold based catalyst (TTIP coated gold foam), no loss of activity during continuous operation at $250{ }^{\circ} \mathrm{C}$ and high $\mathrm{CO}$ concentrations of 12 Vol\% was detected over 24 hours (see Figure 3a).

In conclusion, nanoporous gold used as a scaffold for oxidic nanoparticles, has distinct advantages in comparison to deposited Au nanoparticles. These novel oxide/npAu catalysts show outstandingly high activities at low and high temperatures and good long term stability. Thus, these materials open the door for high temperature applications, such as exhaust gas catalysis, for instance. 


\section{Acknowledgement}

We thank the University Bremen for financial support within the initiative "FuncBand". We gratefully acknowledge the experimental support (SEM) of Petra Witte (Prof. Willems, Historical Geology - Palaeontology, Geology department of the University Bremen). Work at LLNL was performed under the auspices of the US DOE by LLNL under Contract DE-AC52-07NA27344. Project 12-ERD-035 was funded by the LDRD Program at LLNL.

\section{References}

1. C. H. Christensen, J. K. Norskov, Science 327, 278 (Jan, 2010).

2. T. Ishida, M. Haruta, Angew. Chem.-Int. Edit. 46, 7154 (2007).

3. Y. L. Chen, B. L. Zhu, M. Y. Yao, S. R. Wang, S. M. Zhang, Catal Commun 11, 1003 (Jul 1, 2010).

4. Z. W. Wang, X. V. Wang, D. Y. Zeng, M. S. Chen, H. L. Wan, Catal Today 160, 144 (Feb 2, 2011).

5. R. K. Grasselli, Top Catal 21, 79 (Oct, 2002).

6. J. P. P. N. H.-. Nippon Shokubai Co. Ltd., Intern. Pub. No. W097/34692; Dow Chemical Co., Intern. Pub. No. W098/00413, W098/00414, W098/00415; Bayer AG, DE 19804712 A1.

7. J. M. Gottfried, K. J. Schmidt, S. L. M. Schroeder, K. Christmann, Surf Sci 525, 184 (Feb 10, 2003).

8. J. Biener et al., Langmuir 26, 13736 (Sep 7, 2010).

9. A. S. K. Hashmi, G. J. Hutchings, Angew. Chem.-Int. Edit. 45, 7896 (2006).

10. G. J. Hutchings, Catal. Today 100, 55 (Feb, 2005).

11. I. X. Green, W. Tang, M. Neurock, J. T. Yates, Science 333, 736 (August 5, 2011, 2011).

12. J. Erlebacher, M. J. Aziz, A. Karma, N. Dimitrov, K. Sieradzki, Nature 410, 450 (Mar, 2001).

13. A. J. Forty, Nature 282, 597 (1979).

14. D. Lee et al., Scr. Mater. 56, 437 (2007).

15. J. Weissmuller, H. L. Duan, D. Farkas, Acta Mater. 58, 1 (Jan, 2010).

16. C. C. Jia et al., J Phys Chem C 113, 16138 (Sep 10, 2009).

17. S. O. Kucheyev et al., Langmuir 24, 943 (Feb, 2008).

18. T. Yan, J. Gong, C. B. Mullins, J. Am. Chem. Soc. 131, 16189 (Nov, 2009).

19. U. Diebold, Surf. Sci. Rep. 48, 53 (2003).

20. Z. Y. Jiang, Q. Kuang, Z. X. Xie, L. S. Zheng, Adv Funct Mater 20, 3634 (Nov 9, 2010).

21. W. F. Yan et al., J Phys Chem B 109, 10676 (Jun 2, 2005).

22. H. Rath et al., J Appl Phys 105, (Apr 1, 2009).

23. M. M. Biener et al., Nano Lett. 11, 3085 (Aug, 2011).

24. Q. Feng, P. H. Wen, Z. Q. Tao, Y. Ishikawa, H. Itoh, Appl Phys Lett 97, (Sep 27, 2010).

25. A. Haruta, Chem Rec 3, 75 (2003).

26. T. Fujitani, I. Nakamura, Angewandte Chemie International Edition 50, 10144 (2011).

27. A. Wittstock et al., J. Phys. Chem. C 113, 5593 (2009).

28. A. Wittstock et al., J Phys Chem C 113, 5593 (Apr 9, 2009).

29. Y. Denkwitz et al., Catal. Lett. 119, 199 (Dec, 2007).

30. M. M. Schubert et al., J. Catal. 197, 113 (Jan, 2001).

31. V. Aguilar-Guerrero, B. C. Gates, Catal. Lett. 130, 108 (Jun, 2009).

32. D. W. Goodman, M. S. Chen, Chem. Soc. Rev. 37, 1860 (Sep, 2008).

33. G. W. Nyce, J. R. Hayes, A. V. Hamza, J. H. Satcher, Chem. Mat. 19, 344 (Feb, 2007). 
a)

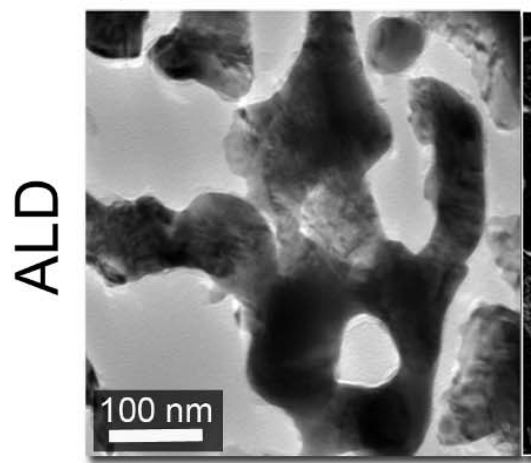

d)

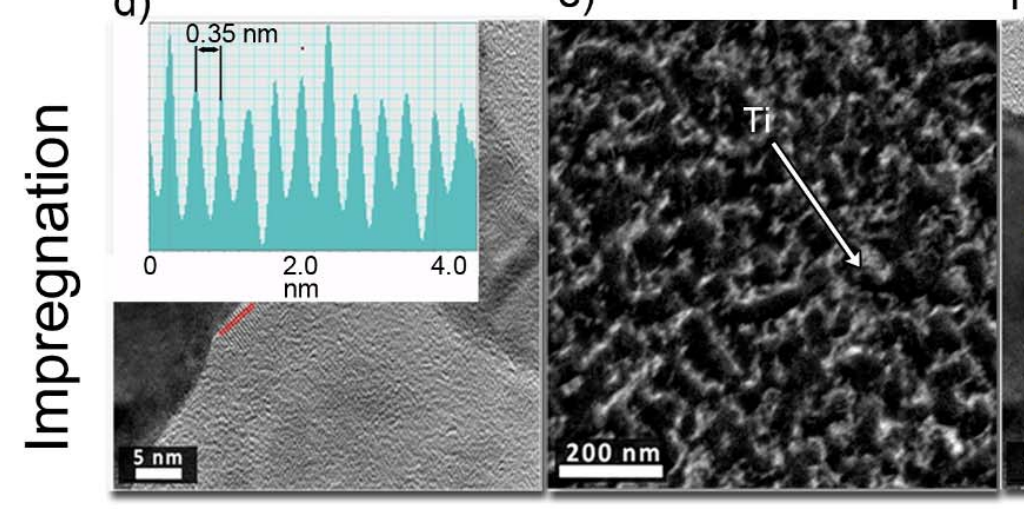

c)

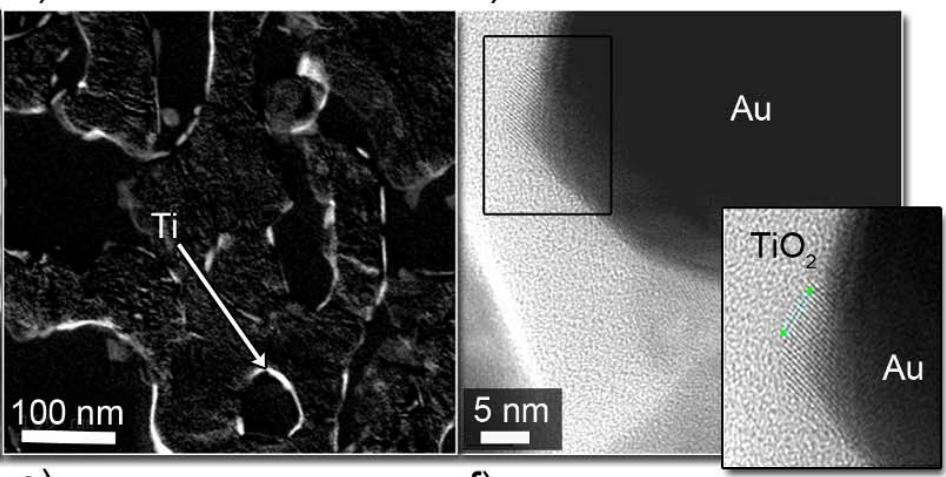

f)

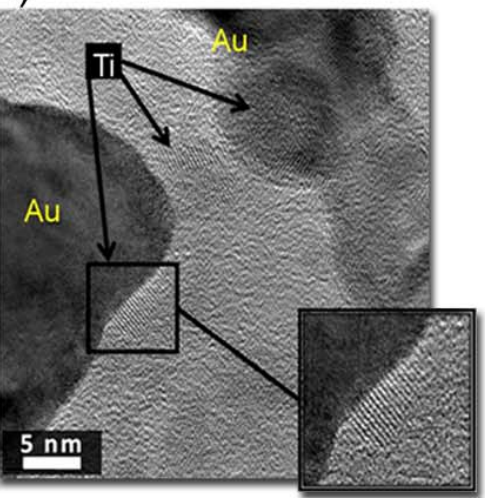

Figure 1: Modification, control over deposition and crystallinity; high resolution transmission electron micrographs (HR-TEM) of Au ligaments with titania nanoparticles deposited by ALD (a-c:particle size $\sim 10 \mathrm{~nm}$ in diameter) and TTIP impregnation (d-f: particle size $\sim 5 \mathrm{~nm}$ in diameter); $(b+e)$ Titanium map obtained by energy filtered TEM proving the very good dispersion of the TiOx particles in the porous structure (Ti represented by bright color); (d) determination of the largest lattice fringe distance by measurement of lattice fringe intensity along a line; the value of $0.36 \mathrm{~nm} \pm 0.01 \mathrm{~nm}$ fits to $\{101\}$ lattice planes of anatase and is in a good agreement with the literature $(0.35 \mathrm{~nm}) .(24)$ 
a)

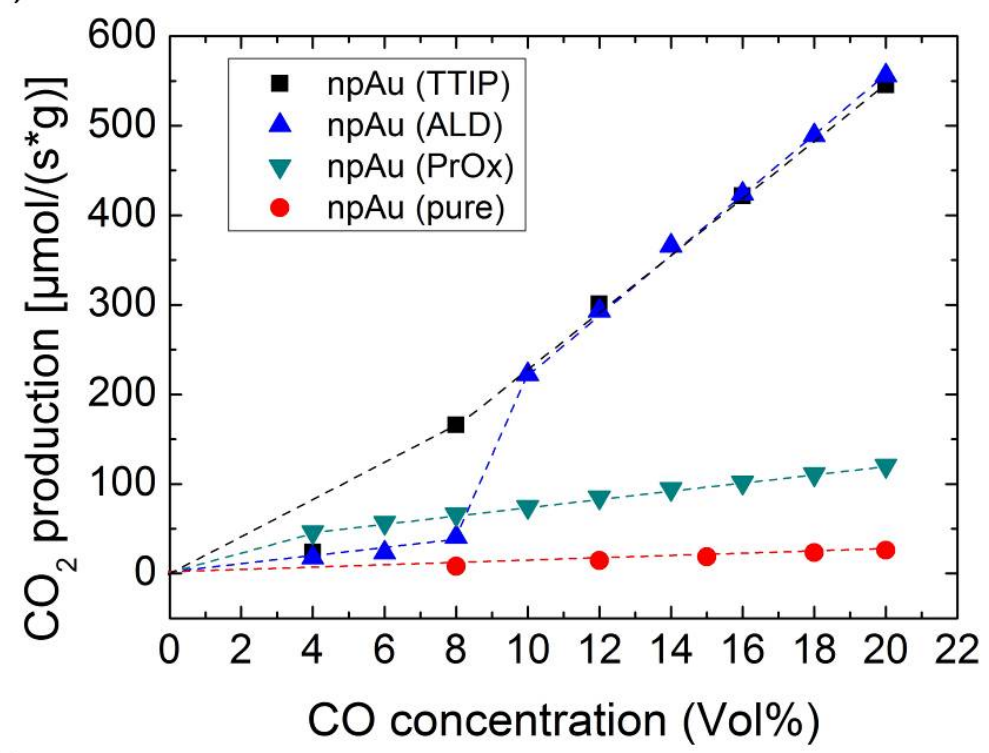

b)

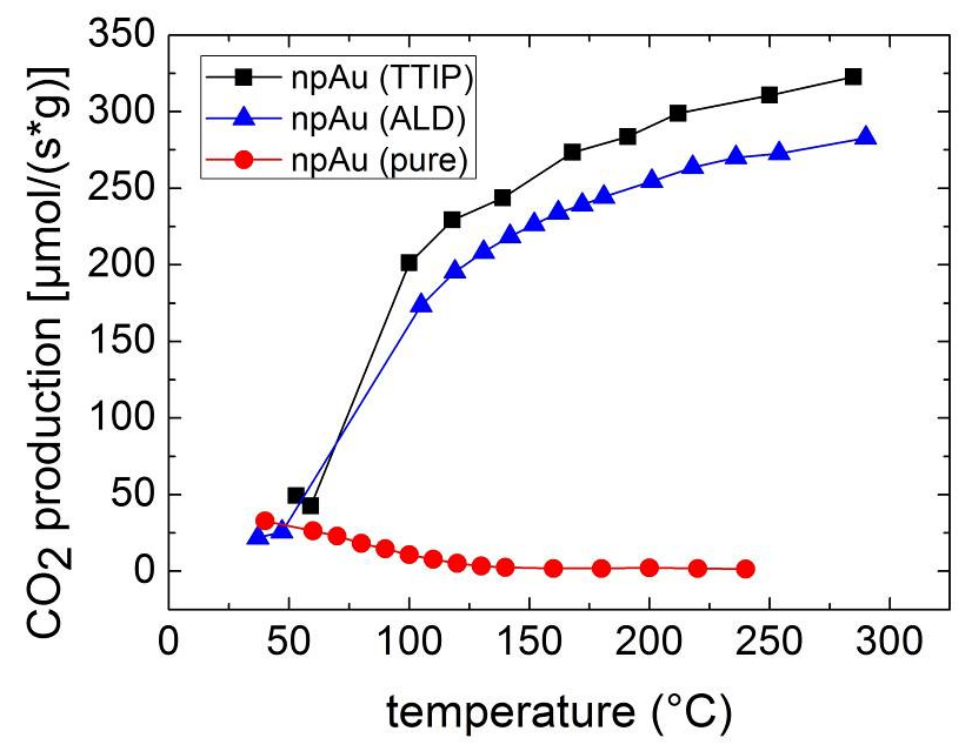

Figure 2: Catalytic activity; (a) activity of pristine np-Au in comparison to PrOx and TiOx (TTIP and ALD) modified np-Au samples at $60{ }^{\circ} \mathrm{C}$ reactor temperature for varying $\mathrm{CO}$ concentrations (30 vol\% $\mathrm{O}_{2}$ ); (b) activity of a pure npAu sample and TiOx coated npAu samples (TTIP and ALD) as a function of the sample temperature (12 vol\% CO, 30 vol\% O2, $\mathrm{He}$ as carrier); please note that the sample temperature is generally higher than the reactor temperature due to the strongly exothermic reaction (see Figure S2 for a detailed correlation). For example the reactor temperature of $60^{\circ} \mathrm{C}$ in section (a) corresponds to a sample temperature of about $110^{\circ} \mathrm{C}$. 


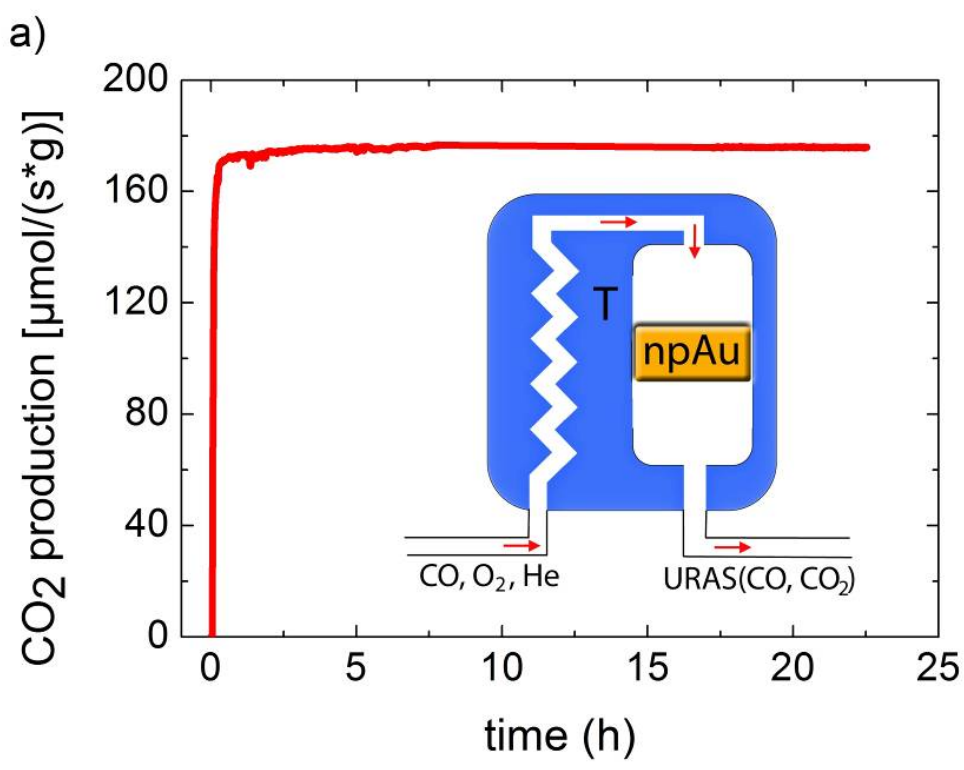

b)

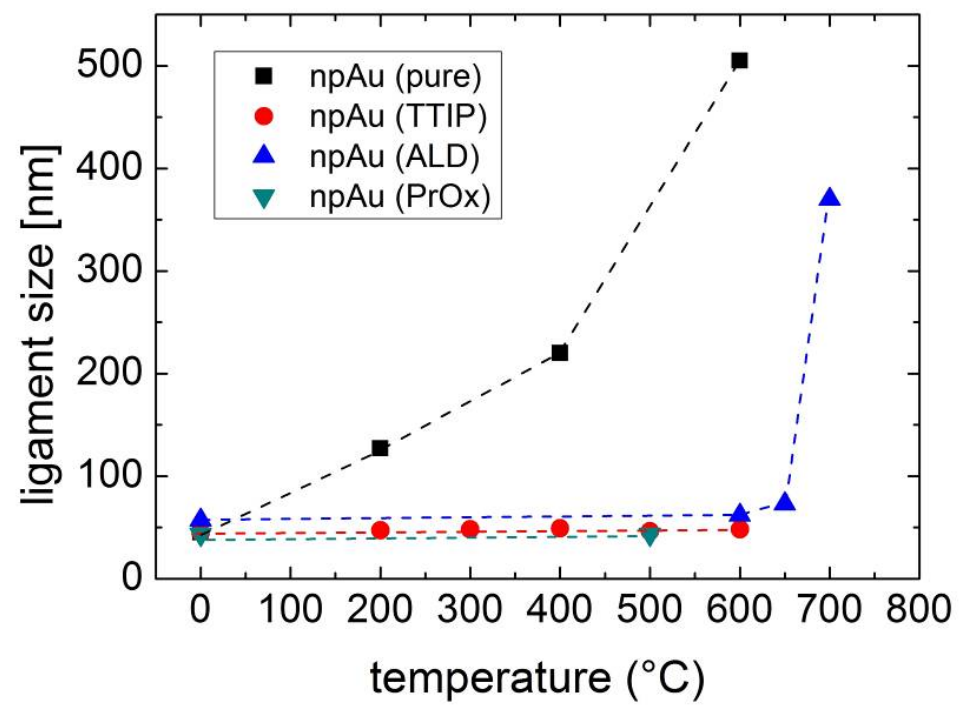

Figure 3: Catalytic long term stability and thermal stability of the catalysts; (a) long-term stability of a TiOx functionalized npAu (TTIP) at $250{ }^{\circ} \mathrm{C}$ and $12 \mathrm{vol} \% \mathrm{CO}$; the activity is stable over 24 hours; the inset shows a scheme of the used reactor; (b) thermal stability of pristine npAu in comparison to PrOx and TiOx (TTIP and ALD) modified npAu samples; whereas the ligament size of the pure npAu is increased due to coarsening with increasing temperature, the oxide deposits on the gold ligaments (PrOx and TiOx modified samples (ALD and TTIP)) stabilize the structure up to $500{ }^{\circ} \mathrm{C}$ for praseodymia and $600{ }^{\circ} \mathrm{C}$ in case of TiOx. 